\title{
La práctica docente reflexiva en profesores mexicanos ante los retos de la nueva ciudadanía
}

\section{Reflective teaching practice in mexican teachers facing the challenges of a new form of citizenship}

\author{
Cecilia Osuna Lever ${ }^{1}$ \\ cecilia.osuna@cetys.mx \\ Karla María Díaz López \\ karla.díaz@cetys.mx \\ CETYS Universidad, Baja California, México
}

\section{Resumen:}

Los docentes llevan sobre sí el peso de lograr una verdadera formación integral $y_{\text {, }}$ por si fuera poco, deben además entregar reportes administrativos, realizar gestiones escolares, desarrollar investigación o vinculación con diversos sectores, etc. Lo anterior, deja poco tiempo para hacer un alto en la enseñanza a fin de reflexionar sobre ¿cómo lograr realmente que sus estudiantes se involucren en sus aprendizajes? ¿Cómo hacer para que estos tengan sentido para el alumno? El docente debe recapacitar continuamente sobre su labor profesional con objeto de innovarla y mejorarla (Galbán, 2016). Frente al mundo cada vez más deshumanizado, la educación se perfila como el único medio para promover y fortalecer la formación valoral, la conciencia de la dignidad de las personas, así como el servicio a los demás (Patiño, 2015). Por lo tanto, en este trabajo se presentan los resultados de un estudio de

\begin{abstract}
:
Teachers bear the burden of achieving a true integral education and, if that were not enough, they must also submit administrative reports, carry out school management, develop research or establish linkages with various sectors, etc. Do all these tasks keep teachers from reflecting on how to really get their students involved in their learning? How can teachers help students make sense of their own learning? Teachers must continually rethink their professional work in order to innovate and improve (Galbán, 2016). Faced with an increasingly dehumanised world, education is emerging as the only means to promote and strengthen education in values, awareness of the dignity of people as well as service to others (Patiño, 2015). Therefore, this paper presents the results of a case study in which 26 teachers who teach at various levels of the Mexican educational system, did an
\end{abstract}

1 Dirección para correspondencia (correspondence address):

Cecilia Osuna Lever. Directora del Colegio de Ciencias Sociales y Humanidades. Centro de Enseñanza Técnica y Superior, CETYS Universidad. Km 1 Camino a Microondas Trinidad. Fraccionamiento Las Palmas 3ra Sección. Ensenada, B.C., C.P. 22860 (México). 
La práctica docente reflexiva en profesores mexicanos ante los retos de la nueva ciudadanía

Cecilia Osuna lever y Karla María Díaz López

caso en el que 26 profesores que imparten clases en los diversos niveles del sistema educativo mexicano, realizaron un ejercicio de autoobservación y reflexión de su práctica docente, con objeto de detectar cuáles factores están limitando el aprendizaje. El análisis de las observaciones permitió identificar que hay aspectos no necesariamente vinculados a los contenidos, que impiden que los objetivos formativos se logren. En particular, sobresalieron los relacionados con el proceso grupal (motivación, trabajo en equipo e integración) que prevalece en el aula, mismo que podría obstaculizar el fortalecimiento de la formación integral entre los educandos de cara a la nueva ciudadanía.

\section{Palabras clave:}

Práctica docente reflexiva; formación integral; fines educativos; estudio de caso. exercise of self-observation and reflection of their teaching practice, in order to detect which factors are limiting learning. The analysis of the observations made it possible to identify that there are aspects, not necessarily linked to the contents, that prevent the training objectives from being achieved. Specifically, those aspects are related to group process (motivation, team work and integration) and they might hinder the strengthening of comprehensive training among students.

\section{Key words:}

Reflective teaching practice; integral education; educational purposes; case study.

\section{Résumé:}

Les enseignants ont portent le poids de la réalisation d'une véritable éducation intégrale et, si cela ne suffisait pas, ils doivent également fournir des rapports administratifs, assurer la gestion de l'école, développer des recherches ou établir des liens avec divers secteurs, etc. Ce qui précède laisse peu de temps pour faire une pause et réfléchir à la façon $d^{\prime}$ impliquer réellement les élèves dans leur apprentissage? Comment les rendre compréhensibles à l'étudiant? L'enseignant doit continuellement repenser son travail professionnel pour l'innover et l'améliorer (Galbán, 2016). Face à un monde de plus en plus déshumanisé, l'éducation apparaît comme le seul moyen de promouvoir et de renforcer l'éducation à la valeur, la conscience de la dignité des personnes ainsi que le service aux autres (Patiño, 2015). Par conséquent, cet article présente les résultats d'une étude de cas dans laquelle 26 enseignants qui enseignent à différents niveaux du système éducatif mexicain, ont fait un exercice d'auto-observation et de réflexion de leur pratique d'enseignement, afin de détecter les facteurs qui limitent l'apprentissage. L'analyse des observations a permis d'identifier qu'il existe des aspects pas nécessairement liés aux contenus qui empêchent d'atteindre les objectifs de formation. En particulier, ceux qui se rapportent au processus de groupe (motivation, travail d'équipe et intégration) qui prévaut dans la salle de classe se démarquent, ce qui pourrait entraver le renforcement d'une formation complète parmi les étudiants.

\section{Mots clés:}

Pratique de I'enseignement réflexif; éducation intégrale; objectifs pédagogiques; étude de cas.

Fecha de recepción: 1-6-2018

Fecha de aceptación: 15-9-2018 


\section{Introducción}

Hablar sobre los fines de la educación puede ser tan diverso como culturas hay en el mundo, pues estos no son universales sino que suelen depender de los intereses, la visión, costumbres, economía y prospección de cada país. La relevancia de la educación es que humaniza a las personas. A decir de Delval (1999) la educación "[...] da al hombre inmensas oportunidades de aprender y le ha permitido construir una cultura, y la cultura es la que hace al hombre ser hombre" (p.1). No obstante, en diversos países la educación actualmente está en crisis, pues se siguen reproduciendo modelos de antaño anclados en la transmisión de conocimientos desarticulados y repetitivos, en ocasiones demasiado técnicos y que no han evolucionado aparejados al contexto globalizado actual, caracterizado por la abundante información y el acelerado desarrollo tecnológico de vanguardia.

Delval subrayó que debemos educar para formar individuos capaces de construir representaciones adecuadas del ambiente, que reflexionen sobre los fenómenos sociales, culturales, económicos y naturales de su entorno y sobre su propia conducta, para que pueda "ser un hombre más libre" (1999, p. 102). Que plasmen en su conducta valores de justicia, respeto, colaboración, solidaridad y búsqueda del bien común, a fin de transformar las sociedades hacia modelos de interacción más justos y equitativos.

En la actualidad, la educación de calidad debe orientarse a que los educandos adquieran aprendizajes significativos que les resulten de utilidad para resolver problemas de la vida cotidiana. A pesar de que los docentes suelen diseñar e implementar planeaciones didácticas y acercan los recursos físicos y tecnológicos a sus estudiantes, tienden a desconocer los diversos factores o elementos que intervienen en los procesos de enseñanza-aprendizaje.

En particular, el sistema educativo mexicano ha enfrentado una severa crisis que se ha gestado a lo largo de seis décadas (1940-2000) y que se ha traducido en un estancamiento en los niveles de rendimiento académico de los estudiantes, en un desprestigio social del docente, infraestructura con graves deterioros, atraso en equipamiento tecnológico, etc., lo que ha llevado a una "descomposición operativa y moral del sistema educativo" (Guevara, 2016, p. 21). Uno de los aspectos más destacados esta crisis, es que no se está formando personas reflexivas y 
críticas, competentes para dar solución a problemáticas de la vida cotidiana, con valores y actitudes necesarios como el trabajo en equipo y la colaboración, a fin de que impacten positivamente en el desarrollo futuro de la nación.

A su vez, los resultados obtenidos por los estudiantes mexicanos en pruebas como PISA (Programme for International Student Assessment, por sus siglas en inglés), aplicada por la Organización para la Cooperación y Desarrollo Económico (OCDE, 2016), ENLACE (Evaluación Nacional del Logro Académico en Centros Escolares 2006-2013) y PLANEA (Plan Nacional para la Evaluación de los Aprendizajes 2015-2017), estas dos últimas se aplican en México, consistentemente confirman los bajos niveles en el logro de los aprendizajes esperados. Por ejemplo, en Matemáticas los jóvenes mexicanos no son capaces de resolver problemas que impliquen el uso del pensamiento geométrico, aplicación de ecuaciones lineales o de ubicación espacial, entre otros, asimismo, en disciplinas como Lengua y Ciencias los resultados son muy semejantes. Se observa una dicotomía en esta problemática, pues el profesor tiene injerencia directa en estos resultados, pero en ocasiones él se percibe ajeno a esta problemática. A decir de Guevara (2016), "muchos profesores no se habitúan todavía a la tarea de reflexionar de forma crítica y sistemática sobre su práctica cotidiana, ni buscan el auto-perfeccionamiento" (p. 49). Por lo que es necesario que el docente contribuya en el mejoramiento de los niveles educativos en los estudiantes mexicanos, analizando críticamente su actuación en el aula y tratando de enfocar sus esfuerzos en la mejora de su actividad docente, con objeto de contribuir al logro de aprendizajes significativos y el cumplimiento de los fines educativos.

Cabe referir que en marzo de 2017, la Secretaria de Educación Pública (SEP) presentó el nuevo Modelo Educativo Mexicano, en este se contempla que la Educación Básica y Media Superior pública contribuirán en la formación de ciudadanos libres, participativos, responsables e informados, capaces de ejercer y defender sus derechos y que participen activamente en la vida social, económica y política de México (SEP, 2016). Asimismo, se establece la re-organización del Sistema Educativo en cinco ejes: 1. Planteamiento Curricular, basado en un enfoque humanista, se compromete el desarrollo de aprendizajes clave que contribuyan al desarrollo integral de los estudiantes y que les permiten aprender a lo largo de la vida, también se enfatiza el desarrollo de las habilidades socioemocionales, se otorga autonomía curricular a las escuelas para 
adaptar contenidos educativos a las necesidades y contextos específicos de sus estudiantes. 2. La escuela al centro del Sistema Educativo, se pretende transitar de un sistema educativo organizado de manera vertical a uno horizontal, con objeto de lograr escuelas con mayor autonomía de gestión: a) plantillas de maestros y directivos fortalecidos, b) liderazgo directivo, c) trabajo colegiado, d) menor carga administrativa, e) infraestructura digna y acceso a las TIC con buena conectividad, f) presupuesto propio, g) asistencia técnico pedagógica de calidad y h) mayor participación de los padres y madres de familia. 3. Formación y desarrollo profesional docente, el profesor debe comprometerse con la mejora constante de su práctica docente y será capaz de adaptar el currículo a su contexto específico centrándose en el aprendizaje de sus estudiantes y en la generación de ambientes de aprendizaje incluyentes. Mientras que en el eje 4 y 5 se refieren a la inclusión y equidad así como la gobernanza del Sistema Educativo respectivamente (SEP, 2017). De acuerdo con Osuna y Díaz (2017) a través de este nuevo Modelo el gobierno mexicano intenta formar ciudadanos reflexivos y críticos y apuesta de manera importante por el quehacer profesional docente, a quien se le concibe como un profesional que cuenta con recursos pedagógicos innovadores, que desarrolla sus conocimientos, habilidades, actitudes y valores necesarios para llevar a cabo un trabajo planeado y orientado al aprendizaje de los estudiantes y a la solución a los problemas diarios y diversos que se presentan en el aula, y que retoma métodos actualizados de enseñanzaaprendizaje (SEP, 2017)

\section{Marco Teórico}

La docencia es una vocación, un "llamado" que compromete y que en muchos casos da sentido a la existencia misma del profesor y se extiende hasta la del educando. Ortega y Gárate (2017), señalaron que "para educar hay que ser un experto en humanidad, tener entrañas de compasión hasta hacer nuestra la situación del otro, hacerse cargo de él" (p. 26), referido el "otro" al educando. Esta frase implica un alto grado de compromiso y responsabilidad que obliga al docente a responder por el otro, a ser sensible a su necesidad, es una "pasión". Esto implica el actuar responsable del profesor referido a todos los ámbitos que involucran a la docencia. 
El desarrollo profesional docente hace referencia a procesos mediante los cuales el profesorado mejora su repertorio de capacidades profesionales (Marcelo y Vaillant, 2009). Al respecto Barandiaran, Muela, López de Arana, Larrea y Martínez (2015) propusieron que este se sustente en la reflexión y el análisis de la práctica profesional como estrategia de trabajo conjunto:

La formación debe proponer un proceso que capacite al profesorado para aprender a aprender, pero también para aprender a desaprender en comunicación, autoanálisis y regulación propia, mediante conocimientos, destrezas y actitudes a fin de desarrollar profesionales inquietos e innovadores que aprendan de sus aciertos y errores" (p. 123).

Por su parte, Araujo y Martins de Araujo (2005) expresaron que es necesario entender la formación docente como un proceso que consiste en construir conocimientos y teorías sobre la práctica docente a partir de la reflexión crítica.

Según Dewey (citado en Bárcena 2005), el pensamiento reflexivo tiene dos etapas: a) estado de duda o perplejidad que desencadena una indagación deliberativa con objeto de solucionar algún problema; y b) proceso operativo de investigación que permita obtener elementos concretos para solucionar el problema, aquí finaliza la elaboración de juicios para tomar una decisión concreta y se actúa para modificar la problemática detectada, otorgando así, a los acontecimientos educativos, un alto grado de significado. Según Dewey (citado en Bárcena, 2005), el docente debe tener la actitud ética necesaria para activar el proceso reflexivo, es decir, abrir la mente, reconocer la posibilidad de error y ofrecer una escucha activa sobre lo que derive de ello. Se requiere una dosis de humildad para reconocer que la práctica docente puede mejorar y por ende, redituar en mejores aprendizajes en nuestros estudiantes. Otra actitud imprescindible en el docente, es el entusiasmo como una especie de motivación interna, que permitirá la aplicación de determinadas estrategias mentales con objeto de mejorar la habilidad de su ejecución dentro del mismo proceso y la última actitud es la responsabilidad intelectual que le permita asumir las consecuencias originadas por los pasos proyectados en las decisiones tomadas.

Por lo tanto, es deber de los docentes comprometerse a hacer bien su trabajo y reflexionar continuamente sobre lo que pueden mejorar. 
En este sentido, la enseñanza reflexiva permite analizar la propia labor docente, con objeto de dinamizarla y mejorarla (Galbán, 2016), pero referida esta a una postura permanente, asociada a un análisis de la actuación docente tal como lo apuntó Bárcena (2005) "en educación, la reflexión conlleva la connotación de hacer deliberaciones, elecciones, tomar decisiones sobre cursos alternativos de acción" (p. 147). Esto necesariamente implica una racionalidad del juicio que involucra una crítica razonada a una situación dada y que debe traducirse en un cambio, es decir, en una modificación de la enseñanza basada en un juicio de naturaleza pedagógica.

En el mismo orden de ideas, en su momento, Freire (2004) enfatizó que es necesario que los profesores reflexionen sobre su práctica pedagógica de modo permanente, en el marco de un análisis sociopolítico y económico-cultural más amplio. Ello implica que la competencia pedagógica del educador no se limita a sus conocimientos teóricos disciplinares y didácticos, sino que, además, exige tener una visión y postura frente al mundo y frente al contexto real en el que se está dando la acción educativa. Para este autor la práctica docente crítica, "[...] encierra el movimiento dinámico, dialéctico, entre el hacer y el pensar sobre el hacer" (Freire, 2004, p. 16). Por lo tanto, la práctica debe ser analizada, desmenuzada, criticada a la luz de las teorías y de la misma experiencia en un proceso dialéctico y dinámico, que genere nuevos conocimientos y nuevas prácticas. Así, la curiosidad se va volviendo crítica y capaz de promover cambios reales, sin duda en el pensamiento de Paulo Freire, la reflexión y la curiosidad epistemológica están intrínsecamente relacionadas con su conceptualización de la práctica docente (Rodrigues, 2013).

Philippe Perrenoud también ha contribuido en el entendimiento de la práctica docente reflexiva, ya que en principio describió los procesos mentales implicados en la práctica reflexiva, para lo cual, en primera instancia realizó una distinción entre la reflexión en la acción, misma que implica reflexión durante el proceso, sobre la situación vivida: "consiste en preguntarse por qué pasa lo que pasa o va a pasar, lo que podemos hacer, cuál es la mejor táctica, qué orientaciones y qué precauciones hay que tomar, qué riesgos existen, etc." (Perrenoud, 2004, p. 30) y la reflexión sobre la acción realizada, en esta se concibe la acción como un objeto de reflexión, posterior a su realización, para comprender y analizar lo que se hizo, si se podría haber hecho otra cosa, qué se debería hacer para mejorar la acción o los resultados a alcanzar. En 
palabras de Rodrigues (2013) conlleva crítica, análisis, comparación con otras situaciones similares y compilación de evidencias, lo cual conduce a la toma de decisiones.

Asimismo, Perrenoud observó que durante la acción pedagógica resulta difícil reflexionar pausadamente, por el ritmo constante y acelerado de toma de decisión y de acción. No obstante, fuera de la acción pedagógica, el profesor puede reflexionar sobre lo que ha pasado en clase, los resultados de su acción, los problemas enfrentados y la selección de otras estrategias de enseñanza (Rodrigues, 2013). La reflexión es "retrospectiva y prospectiva y conecta el pasado con el futuro" (Perrenoud, 2004, p. 35). La retrospección puede contribuir a "capitalizar la acción" y transformarla en conocimientos que pueden ser utilizados en otras circunstancias. La prospección puede aportar a la planificación de nuevas actividades, la repetición de experiencias exitosas o la anticipación de problemas que puedan ocurrir en el transcurso de la práctica (Rodrigues, 2013).

Así pues, la práctica docente reflexiva busca potenciar la capacidad del profesor para analizar continuamente sobre su quehacer y convertir esta capacidad de la inteligencia en un hábito profesional que lo acerque a la realidad del aula (Galbán, 2016), de esta manera puede hacer adecuaciones al proceso de enseñanza-aprendizaje, así como seleccionar y aplicar estrategias didácticas que le permitan potenciar el logro de aprendizajes significativos en sus estudiantes. En este orden de ideas, Sánchez, Lara, Bravo y Navales (2015) han referido que, en la reflexión de la propia práctica docente, el profesor debe utilizar los conocimientos y pensamientos y contrastarlos con los aspectos normativos y con los resultados de su modo de actuación profesional.

Lo antes dicho, deja en claro que el uso de estrategias didácticas adecuadas potencian el logro de aprendizajes significativos, pero no nos referimos solo a contenidos sino también, a la parte formativa de los alumnos, pues al educar se promueven valores como la libertad, tolerancia, respeto, responsabilidad, entre otros; valores morales que expresan lo que los estudiantes consideran importante en sus vidas, es decir, las ideas que existen detrás del comportamiento y que se aplican en la convivencia con los demás. Esta es una manera de transitar a la educación en la ciudadanía, misma que ayuda a los estudiantes a desarrollar su propia identidad y su participación en la sociedad (Veugelers, 2018). Tal como lo apunta Buxarraís (2018), desde la educación y sus cuatro 
pilares: aprender a conocer, aprender a actuar, aprender a ser y aprender a convivir, se deben definir determinados aprendizajes para los estudiantes y determinadas enseñanzas para el profesorado.

Es así como se revela que la práctica docente reflexiva promueve la construcción de conocimientos y debe comprender un fundamento para la planeación didáctica, y reflejarse también en la evaluación del proceso educativo. Debe constituir una actividad cotidiana en la vida docente, pues de ser ejercida habitualmente puede transformar de manera positiva el proceso educativo. ¿Será entonces esta la opción para mejorar algunas habilidades docentes que se traduzcan en el mejoramiento de los índices de aprovechamiento escolar y formación de valores para la ciudadanía en los estudiantes?

\section{Marco empírico}

\section{Diseño de la investigación}

El diseño se enmarcó en los métodos cualitativos, enfocado a un estudio de caso simple (Creswell, 2009). Cuyo objetivo fue definir algunos de los factores que obstaculizan el proceso de enseñanza aprendizaje, por medio de la autoreflexión de la práctica docente, a fin de mejorar la enseñanza y contribuir a la formación integral de los estudiantes.

\section{Participantes}

Se promovió la enseñanza reflexiva con 26 profesores en servicio en diferentes niveles del sistema educativo. El $84 \%$ de ellos son mujeres y el resto hombres. La mayoría de los datos de los diferentes niveles educativos en los que se aplicó la autoreflexión docente, grados y asignaturas reflejados en este estudio, se muestran en la Tabla 1. 
La práctica docente reflexiva en profesores mexicanos ante los retos de la nueva ciudadanía

Cecilia Osuna Lever y Karla María Díaz López

Tabla 1

Nivel educativo, grado y asignaturas

\begin{tabular}{|c|c|c|}
\hline Nivel educativo & Grado & Asignaturas \\
\hline \multirow[t]{2}{*}{$\begin{array}{l}\text { Educación básica, } \\
\text { preescolar }\end{array}$} & $\begin{array}{l}2 \text { do } \\
2 \text { do }\end{array}$ & $\begin{array}{l}\text { Inglés } \\
\text { Español }\end{array}$ \\
\hline & 2do & Matemáticas \\
\hline \multirow{6}{*}{$\begin{array}{l}\text { Educación básica, } \\
\text { primaria }\end{array}$} & 3 ro & Matemáticas \\
\hline & 4to & Matemáticas \\
\hline & 5to & Matemáticas \\
\hline & 3 ro & Español \\
\hline & 4to & USAER \\
\hline & 4to & Español \\
\hline \multirow{5}{*}{$\begin{array}{l}\text { Educación básica, } \\
\text { secundaria }\end{array}$} & $2 \mathrm{do}$ & Formación cívica y ética \\
\hline & 2 do. & Historia \\
\hline & 1ero & Secundaria \\
\hline & 3ro & Formación cívica y ética \\
\hline & 1 ro & Español I \\
\hline $\begin{array}{l}\text { Educación media } \\
\text { superior }\end{array}$ & 3 ro & Química II \\
\hline \multirow{7}{*}{ Educación superior } & 7 mo semestre & Enseñanza de lenguas \\
\hline & 2do semestre & Comunicación avanzada en español \\
\hline & 3er semestre & Taller de comunicación efectiva \\
\hline & 1er semestre & Técnicas básicas de arquitectura \\
\hline & 5to semestre & Enfermería quirúrgica \\
\hline & 2do semestre & Historia de México \\
\hline & 3er semestre & Liderazgo y comunicación asertiva \\
\hline
\end{tabular}

Fuente: Elaboración propia.

Como se aprecia en la tabla 1, las asignaturas objeto de autoreflexión docente abarcan diferentes áreas de conocimiento y engloban a todos los niveles que componen la estructura del sistema educativo mexicano, lo que permitió tener un panorama más amplio que, aunque no es exhaustivo, si es inclusivo.

\section{Instrumentos para la recolección de los datos}

Se recolectó información por medio de un formato de autoobservación docente diseñado especialmente, conformado por las siguientes categorías: a) Nombre del profesor, b) Nombre, grado y nivel educativo de la asignatura, c) Fecha, hora y duración de la observación, d) Número 
de estudiantes, e) Competencia de la asignatura y objetivo de la sesión observada, f) Registro de la observación, g) Descripción de la problemática identificada, h) Dinámica del grupo: ambiente para el aprendizaje, interacción, distractores, i) Infraestructura: espacios para el aprendizaje y materiales de apoyo, j) Aplicación de la planeación: manejo de contenidos y estrategias didácticas, k) Comentarios.

La observación y llenado del formato se hizo durante un periodo sostenido de tiempo (Creswell, 2009) obedeciendo a estos parámetros: 1. La misma materia y el mismo grupo en todas las observaciones realizadas. 2. Mínimo registrar tres observaciones de tres sesiones de la asignatura elegida.

\section{Procedimiento}

El proceso se dividió en tres etapas y el procedimiento se basó en la recomendación que propone Bárcena (2005) para lograr una auto reflexión docente efectiva.

Etapa 1. Estado de duda o perplejidad. Registrando con objetividad las principales problemáticas enfrentadas en su ejercicio pedagógico, mediante la autoobservación sostenida durante mínimo 3 sesiones de clase y registrando lo acontecido.

Etapa 2. Proceso operativo: se analizaron las observaciones en todos los aspectos registrados, identificando el posible origen de las problemáticas detectadas. Se analizaron los aspectos categorizando si se referían a problemas orientados a la tarea o contenidos o bien, problemas referidos al grupo y su dinámica. Con esta información los profesores diseñaron una planeación didáctica basada en estrategias orientadas a su solución.

Etapa 3. Aplicación de la planeación y registro de resultados. En total se generaron 78 observaciones, lo cual representó al 100\% de los datos. El análisis cualitativo de cada una de las observaciones, se realizó con base en la técnica de análisis de contenido, las categorías de análisis son las que conforman el instrumento de observación y se identificaron los fragmentos de texto que refirieran a las problemáticas detectadas, como unidades de análisis. Se diseñó una matriz en la que se capturaron las categorías, frecuencia de aparición y fragmentos de texto. Una vez concluido el análisis se realizó la ponderación de las frecuencias de aparición y se identificaron por orden de importancia, las principales problemáticas señaladas. 


\section{Resultados y discusión}

Con base a los resultados obtenidos se evidenció que la principal problemática enfrentada por los docentes, no se asocia a la complejidad o cantidad de los contenidos a enseñar, sino a factores que afectan la dinámica grupal y que obstaculizan el aprendizaje (72\%). Solo en seis observaciones $(8 \%)$ se identificaron problemáticas orientadas a contenidos o bien a problemas de aprendizaje de algún alumno en específico. Respecto a los factores que pudieran estar ejerciendo influencia en la dinámica de grupo estos se clasifican en siete: Entorno físico, Entorno personal, Entorno social, Entorno tarea, Técnicas, Tácticas y Procesos grupales (Orozco, 2013). Ver tabla 2.

\section{Tabla 2}

Factores que afectan la dinámica de grupo

\begin{tabular}{ll}
\hline Entorno físico & $\begin{array}{l}\text { Infraestructura y factores ambientales en los que se en- } \\
\text { cuentra el grupo }\end{array}$ \\
\hline Entorno personal & $\begin{array}{l}\text { Número de integrantes del grupo, edad, creencias, clase } \\
\text { social u origen étnico }\end{array}$ \\
\hline Entorno social & $\begin{array}{l}\text { Relaciones interpersonales, motivación, apego, homoge- } \\
\text { neidad y heterogeneidad, status }\end{array}$ \\
\hline Entorno tarea & $\begin{array}{l}\text { Actividades de los integrantes, responsabilidades, objeti- } \\
\text { vos y metas. }\end{array}$ \\
\hline Técnicas & $\begin{array}{l}\text { Herramientas de trabajo para lograr cambios en el trabajo } \\
\text { grupal }\end{array}$ \\
\hline Tácticas & Pasos para lograr la conducción adecuada del grupo \\
\hline Procesos grupales & $\begin{array}{l}\text { Liderazgo, comunicación, motivación, integración, mane- } \\
\text { jo de conflictos, solución de problemas, toma de decisio- } \\
\text { nes y trabajo de equipo }\end{array}$ \\
\hline
\end{tabular}

Fuente: Orozco, O. (2006). Conducción de grupos y las estrategias de intervención grupal. Tijuana, México: Ediciones ILCSA. p. 29.

Con base a la clasificación referida, las problemáticas detectadas en este estudio se ubicaron en términos de procesos grupales, destacando principalmente la falta de motivación de los estudiantes para el trabajo en el aula, con una frecuencia de aparición de 30; en segundo lugar dificultades para el trabajo en equipo (24) y en tercero la falta de integración (18). En la tabla 3 se muestra la frecuencia de aparición de los principales procesos grupales detectados. Además, se registraron problemáticas 
referidas a la ausencia de valores deseables para la convivencia, que por supuesto se fomentan de manera natural en los procesos grupales.

Tabla 3

Frecuencia de aparición de los procesos grupales identificados

\begin{tabular}{cll}
\hline & \multicolumn{1}{c}{ Tipo de proceso } & Frecuencia de aparición \\
\hline & Motivación & 30 \\
\cline { 2 - 3 } $\begin{array}{c}\text { Procesos } \\
\text { grupales } \\
\text { identificados }\end{array}$ & Trabajo en equipo & 24 \\
\cline { 2 - 3 } & Integración & 18 \\
\cline { 2 - 3 } & Comunicación & 15 \\
\cline { 2 - 3 } & Liderazgo & 9 \\
\cline { 2 - 3 } Ausencia & Manejo de conflictos en el aula & 3 \\
\cline { 2 - 3 } de Valores/ & Tolerancia & 6 \\
\cline { 2 - 3 } Actitudes & Respeto & 3 \\
\hline
\end{tabular}

Fuente: Elaboración propia con base en los resultados.

La relevancia de estos resultados estriba en evidenciar la importancia que tienen los procesos grupales para el logro de las intenciones formativas, hallazgo que no se esperaba encontrar, por lo cual es significativo que fueran identificados. Los procesos grupales dinamizan al grupo y a través de ellos, el docente puede lograr mejores resultados manejando al grupo mediante técnicas adecuadas. Por ejemplo, lograr la motivación en el alumno es uno de los mayores retos para el docente, dado que si el alumno no siente interés por aprender no se involucrará en el proceso. Respecto al trabajo en equipo sus efectos son benéficos, pues al integrar equipos se supera el desempeño individual que podría darse por cada estudiante, se facilita la participación en decisiones, se promueven valores (responsabilidad, tolerancia, paciencia etc.), aumenta la motivación y genera sinergia positiva por medio del esfuerzo coordinado (Orozco, 2013). Es claro que el uso de estrategias de aprendizaje grupal, es un mecanismo para el fomento de valores para la convivencia.

Por otro lado, la integración grupal no puede soslayarse, esta implica la capacidad de un grupo para formarse en nuevos equipos y funcionar correctamente, involucra respeto y tolerancia a la diversidad de opiniones y a los subgrupos diferentes al propio. También mejora el desempeño y las relaciones interpersonales, entrena en habilidades sociales y de comunicación y promueve valores. A este respecto, el último informe de 
la prueba PISA (OCDE, 2017) sobre el trabajo colaborativo o en equipo, deja notar que este fomenta actitudes más positivas en las relaciones de los estudiantes y les ayuda a trabajar de forma más efectiva y eficiente.

En este mismo sentido, los estudiantes que respondieron la prueba PISA en 2015, indicaron que cuando se trabaja en equipo se toman mejores decisiones que en trabajo individual. Cabe destacar que en dicho informe México quedó en el lugar 44 de 50 países reportados y muy por debajo de Chile, Uruguay y Costa Rica en el ámbito latinoamericano, en el rubro de fomento y efectividad del trabajo colectivo en el aula. Aunque no es posible generalizar los resultados del estudio de caso objeto de este artículo, ni hacer equivalentes los datos de PISA, nos revelan cierta tendencia referida al bajo nivel de trabajo colaborativo efectivo reportado para México.

Como se puede apreciar, no siempre los problemas de aprendizaje se deben a la complejidad de los contenidos, a factores personales o de otra índole; sino que pudieran deberse a procesos grupales. A decir de Díaz-Barriga (2010), el aprendizaje significativo se favorece mediante estrategias de aprendizaje que propicien el deseo de aprender del estudiante. El aprendizaje significativo sucede cuando el docente interviene con una planeación orientada a los aprendizajes esperados, considerando los procesos grupales y aplicando estrategias para lograr las competencias establecidas. Así, la reflexión personal del docente y la autoobservación de su práctica pedagógica, pueden contribuir a mejorar su enseñanza y favorecer los aprendizajes esperados y la promoción de valores para la convivencia personal y ciudadana. En el caso que nos ocupa, problemáticas tan importantes como la falta de motivación hacia el aprendizaje, deben ser consideradas por los profesores al momento de planear su acción pedagógica, de tal forma que se estimule al alumno y el clima escolar, para prepararlo para el logro de los aprendizajes.

Una vez identificado el problema, los profesores planearon su enseñanza con base en estrategias didácticas orientadas al grupo y focalizadas a estos aspectos, aplicaron la planeación y evaluaron cualitativamente el resultado obtenido, elaborando una carpeta de evidencias con su planeación, materiales y algunos comentarios de sus alumnos. Cabe mencionar que los profesores al realizar todo este proceso de autoreflexión demostraron una actitud ética, al mostrar apertura al cambio y humildad para participar del proceso, el entusiasmo para realizar el ejercicio y la responsabilidad intelectual al decidir solventar el problema, diseñando y aplicando las estrategias adecuadas para ello. A continua- 
La práctica docente reflexiva en profesores mexicanos ante los retos de la nueva

ciudadanía

Cecilia Osuna lever y Karla María Díaz López

ción se presentan algunos fragmentos de las opiniones docentes sobre el resultado de este proceso:

"Fue un gran ejercicio de introspección y muy reflexivo para mejorar en situaciones que a veces creemos no tienen solución o creemos que se nos salen de la manos, cuando lo que debemos hacer siempre primero es observar, después diagnosticar y con base en ello, planear, ejecutar y concluir con una retroalimentación que haga que se mejore nuestro trabajo frente a grupo".

"Es necesario realizar una pauta a situaciones que suelen percibirse como un pequeño detalle y considerar la calidad y no la cantidad de la enseñanza que proporcionamos; ya que ello puede implementar un cambio sumamente importante en el alumno no solamente dentro del aula, sino para todo su desarrollo y vida social".

"Tuve la experiencia vivencial de descubrir la aplicación de las observaciones como una forma de retroalimentación y su importancia para mejorar la práctica docente".

En cuanto a la planeación y aplicación de estrategias para abordar la problemática detectada:

"No cabe duda que realizar observaciones para identificar los problemas del grupo es de vital importancia y se logra una trascendencia cuando se emplean los métodos necesarios para combatirlos".

"Me gustó muchísimo y me deja muchísimo esta forma de trabajo que se llevó al idear la estrategia, el ir paso a paso observando las necesidades, pulirlas, observar una vez más, seguir puliendo y finalmente aplicar lo que se delimitó, esto es lo idóneo para que aprenda el estudiante".

"La planeación es una parte fundamental de la práctica docente. Considero que es una herramienta que puede ayudar a que el maestro lleve a cabo una interesante reflexión sobre su propia actividad"

Los comentarios anteriores reflejan el sentido y utilidad que tuvo para estos profesores analizar su propia práctica docente, detectar las problemáticas que les impiden lograr los aprendizajes y solucionarlas a 
través de una planeación pertinente, motivados por su vocación docente y buscando responder por el otro, por el educando. A decir de Patiño (2015) "un verdadero maestro se preocupa por enseñar lo que el alumno necesita y no lo que él sabe" (p. 108).

\section{Conclusiones y Limitaciones}

En principio, cabe mencionar que a través de este estudio se aporta evidencia que da cuenta que no todas las problemáticas educativas se asocian a la complejidad de los contenidos o de la información que debe aprender el alumno, sino a los procesos por los que atraviesa el grupo, por lo que es imprescindible que se identifiquen dichos procesos.

Al garantizar procesos grupales adecuados como la motivación, la integración y el trabajo en equipo, se promueven además de aprendizajes, la práctica de valores para la convivencia social, lo cual conduce a la formación integral de cara a la nueva ciudadanía. Es trascendental que el docente se detenga a pensar y analizar juiciosamente en lo que está haciendo en su práctica y que registre que resultados está obteniendo, con objeto de implementar innovaciones o ajustes y asegurar en mayor medida el logro de los aprendizajes comprometidos en los currículos escolares. Por ende, la forma en la que se les presentan los contenidos a los estudiantes por medio de estrategias didácticas adecuadas, es igual de relevante que los contenidos por aprender.

La práctica docente reflexiva es clave para la profesionalización de la enseñanza, por lo que se debe buscar ejercitarla constantemente y de forma sistemática, ya que puede ser un factor que contribuya en gran medida a lograr los fines educativos, buscando formar ciudadanos competentes, que resuelvan problemas y que practiquen valores como la justicia, tolerancia, respeto y que sepan trabajar colaborativamente por el bien de su comunidad a fin de transformarla, lo que ajusta perfectamente al modelo de nueva ciudadanía.

Si partimos del referente planteado en el Nuevo Modelo Educativo Mexicano, el Eje 3 Formación y desarrollo profesional docente, sostiene que el profesor debe comprometerse con la mejora de su práctica, un ejercicio periódico como la autoreflexión docente podría contribuir a ello. Por otro lado, respecto al fomento del trabajo en equipo y los procesos grupales, el Eje 1 Planteamiento curricular, se concentra en el desa- 
rrollo de aprendizajes clave que contribuyan al desarrollo integral de los estudiantes y que les permiten aprender a lo largo de la vida y enfatiza el desarrollo de las habilidades socioemocionales, estas cualidades se fomentan a través de los procesos grupales. He ahí la importancia de que los docentes reflexionen concienzudamente sobre su quehacer y la forma de mejorarlo, para contribuir así al logro de los fines educativos.

Este estudio de caso no busca la generalización de los resultados, no obstante, contribuye en la línea de investigación centrada en la práctica docente reflexiva. Asimismo, los lectores que se identifiquen con los haIlazgos presentados, podrán relacionarlos con lo que sucede en sus propios contextos y efectuar las validaciones correspondientes. Finalmente, y en torno a la prospectiva de estos hallazgos, resulta interesante observar como un ejercicio como la autoreflexión puede ampliar el panorama de un docente, al hacerlo consciente de sus limitaciones y mejorarlas, por lo que investigaciones educativas sobre este tema deberían ser promovidas con más regularidad, sobre todo en México, pues los resultados obtenidos podrían apoyar al seguimiento que se debe dar a la implementación y consecución del Nuevo Modelo Educativo y por consecuencia mejorar los niveles de aprovechamiento escolar de los niños mexicanos.

\section{Referencias}

Araujo, E. M. y Martins, C. (2005). Reflexão em Paulo Freire: uma contribuição para a formação continuada de professores. V Colóquio Internacional Paulo Freire, Recife, 19 a 22, setembro 2005.

Barandiaran, A., Muela, A., López, E., Larrea, I. y Martínez, A. (2015). Programa para la mejora de la sensibilidad (PMS) y habilidades de interacción del profesorado de Educación Infantil. Educatio Siglo XXI, 33, 281-302.

Bárcena, F. (2005). La experiencia reflexiva en educación. España: Paidós.

Educación moral y cívica: una pedagog En R. Mínguez y E. Romero (Coords.). La educación ciudadana en un mundo en transformación: miradas y propuestas (pp. 121-129). España: Octaedro Editorial.

Creswell, J. (2009). Research Design. Qualitative, Quantitative and Mixes Methods Approaches. USA: Sage.

Delval, J. (1999). Los fines de la educación. México: Siglo XX.

Díaz-Barriga,A. (2010). Estrategias docentes para un aprendizaje significativo. México: McGraw-Hill.

Freire, P., (2004). Pedagogía de la Autonomía: saberes necesarios para la práctica educativa. São Paulo: Paz e Terra.

Galbán, S. (2016). Hacia una enseñanza reflexiva. México: Trillas. 
La práctica docente reflexiva en profesores mexicanos ante los retos de la nueva ciudadanía

Cecilia Osuna Lever y Karla María Díaz López

Guevara, G. (2016). Poder para el maestro poder para la escuela. México: Ediciones Cal y Arena.

Imbermón, F., (2007). La formación permanente del profesorado. Nuevas ideas para formar en la innovación y en el cambio. Grao: Barcelona.

Marcelo, C. y Vaillant, D. (2009). Desarrollo profesional docente ¿Cómo se aprende a enseñar? Madrid: Narcea.

Organización para la Cooperación y Desarrollo Económico (OCDE). (2016). PISA 2015. Resultados clave. París: Autor. Recuperado de https://www.oecd.org/pisa/pisa2015-results-in-focus-ESP.pdf

Organización para la Cooperación y Desarrollo Económico (OCDE). (2017). PISA 2015. Volumen V Collaborative Solving Problems Summary. París: Autor. Recuperado de http:// www.oecd-ilibrary.org/docserver/download/5dc0aea7-es.pdf?expires $=1516242$ 118yid=idyaccname=guestychecksum=3400E3D55827ACB251C51BA4AB544F21

Orozco, O. (2006). Conducción de grupos y las estrategias de intervención grupal. Tijuana, México: Ediciones ILCSA.

Ortega, P. y Gárate, A. (2017). Una escuela con rostro humano. México: Instituto Educativo del Noroeste AC.

Osuna, C., Díaz, K. y López, M. (2017). El nuevo modelo educativo mexicano 2016. Encuentros y desencuentros. En M. Martin y E. Sebastián (Coords). Hacia un nuevo modelo educativo de calidad y transformador (pp. 41-50). España: Fundación SantiIlana y Universidad de Alcalá.

Patiño, H. (2015). ¿Qué hacen los docentes de excelencia? Claves para la formación humanista en la universidad. México: Universidad Iberoamericana.

Perrenoud, P. (2004). Desarrollar la práctica reflexiva en el oficio de enseñar. Barcelona: Graó.

Rodrigues, R. (2013). El desarrollo de la práctica reflexiva sobre el quehacer docente, apoyada en el uso de un portafolio digital, en el marco de un programa de formación para académicos de la Universidad Centroamericana de Nicaragua. (Tesis Doctoral, Universidad de Barcelona, España) Recuperado de http://diposit.ub.edu/dspace/bitstream/2445/43124/5/RRODRIGUES_TESIS.pdf

Sánchez, R., Lara, L., Bravo, G. y Navales, M. (2015). La autopreparación y la reflexión de la práctica docente: Binomio indispensable en la formación Pedagógica del profesor Universitario. Revista de Cooperación y Bienestar Social, 7, 33-47. Recuperado de http://revistadecooperacion.com/numero7/numero7.pdf \#page=33

Secretaria de Educación Pública. (2017). Antecedentes del Nuevo Modelo Educativo [Sitio web]. Recuperado de https://docs.google.com/gview?url=http://www.gob.mx/ cms/uploads/ attachment/file/207247/ANTECEDENTES.pdf

Secretaria de Educación Pública. (2016). Los fines de la educación en el siglo XXI [Sitio web]. Recuperado de https://www.gob.mx/cms/uploads/attachment/file/114503/ Los_Fines_de_la_Educacio_n_en_el_Siglo_XXI.PDF

Veugelers, W. (2018). La educación moral y para la ciudadanía en el siglo XXI. En R. Mínguez y E. Romero (Coords.). La educación ciudadana en un mundo en transformación: miradas y propuestas (pp. 121-129). España: Octaedro Editorial. 